Archives de sciences sociales des religions

140 | octobre - décembre 2007

Varia

\title{
Le Diaconat permanent. Actes du colloque de 2004, Lyon
}

Paris, Éditions du Cerf, 2007, 368 p.

Nicolas de Bremond d'Ars

\section{OpenEdition}

Journals

Édition électronique

URL : http://journals.openedition.org/assr/11753

DOI : 10.4000/assr. 11753

ISSN : $1777-5825$

Éditeur

Éditions de l'EHESS

Édition imprimée

Date de publication : 1 décembre 2007

ISBN : 978-2-7132-2145-3

ISSN : 0335-5985

Référence électronique

Nicolas de Bremond d'Ars, "Le Diaconat permanent. Actes du colloque de 2004, Lyon », Archives de sciences sociales des religions [En ligne], 140 | octobre - décembre 2007, document 140-66, mis en ligne le 02 juillet 2008, consulté le 21 septembre 2020. URL : http://journals.openedition.org/assr/ 11753 ; DOl : https://doi.org/10.4000/assr.11753

Ce document a été généré automatiquement le 21 septembre 2020.

(c) Archives de sciences sociales des religions 


\title{
Le Diaconat permanent. Actes du colloque de 2004, Lyon
}

Paris, Éditions du Cerf, 2007, 368 p.

\author{
Nicolas de Bremond d'Ars
}

1 L'ouvrage reproduit les actes d'un colloque organisé par la Faculté de Théologie de l'Université Catholique de Lyon les 19-21 novembre 2004. Face à la théologie, l'histoire et la sociologie sont convoquées pour poser des approches complémentaires. La dernière partie offre des réflexions et témoignages.

2 Les études sur le diaconat sont peu abondantes, l'ouvrage comble donc une lacune importante dans la connaissance de ces nouveaux acteurs religieux du catholicisme. L'interdisciplinarité, solidement assurée par de très bonnes contributions, rend ce colloque particulièrement opportun pour le sociologue du catholicisme, certes, mais également pour les chercheurs qui s'intéressent à l'innovation institutionnelle en matière de religion.

D'emblée, avant même la présentation exhaustive, nous pointerons deux questions centrales. La première concerne sans doute l'histoire au premier chef : est-il possible, et à quel prix, après avoir mis entre parenthèses pendant un millénaire cette institution ecclésiastique (les diacres), de bâtir à nouveau une institutionalité religieuse qui vaille pour la modernité? Question qui interroge aussi le théologien Bernard Sesboüé. Deuxièmement, en supposant que cette « rénovation » soit possible, qu'annonce-t-elle d'un nouveau rapport du catholicisme à la modernité sociale? Ces questions ne sont pas abordées directement dans les diverses contributions, mais elles affleurent et soustendent clairement certaines (au moins pour la première).

4 Quant au sujet du colloque : le diaconat existe maintenant depuis trente ans en France. Il serait vain de nier sa place, d'autant que l'effet de ciseau entre la baisse prononcée du nombre de prêtres (environ douze mille en 2007, dont quatre mille six cents de moins de 65 ans) et l'augmentation régulière de la présence des diacres (environ deux mille en 2007) lui donne une visibilité de plus en plus populaire. Cependant, comme le fait remarquer Bernard Sesboüé, il faut compter plutôt deux générations pour juger de la réussite d'un enracinement institutionnel. Le temps long de l'histoire croise ici la 
pertinence sociale contemporaine pour une mise en perspective cohérente. Le colloque n'élude pas cet aspect.

5 L'histoire ouvre les contributions du livre. Bruno Dumons retrace l'apparition du thème diaconal en France (dans le célèbre France, pays de mission des abbés Daniel et Godin en 1943), en Allemagne, avec notamment Josef Hornef, laïc engagé, avec un article publié en 1949 (un ouvrage suivra huit ans plus tard, aussitôt traduit en français), et le courant issu de la Caritas, qui réfléchit au diaconat dès 1951 (cercle d'aspirants au diaconat à Fribourg). La préoccupation se diffuse dans les pays de langue germanique et francophone. Paul Winninger, à Strasbourg, se fait passeur du monde germanique en France, et oriente définitivement les recherches, avec Vers un renouveau du diaconat de 1958, vers l'ecclésiologie. La question est publiquement évoquée à Rome, et le pape Pie XII la reçoit avec prudence, appelant à un travail de maturation théologique et patristique. Karl Rahner, le grand théologien allemand, publie avec l'aide des meilleurs spécialistes une somme monumentale sur le diaconat pour l'ouverture du concile Vatican II, en octobre 1962. L'auteur suit les publications en France, en Allemagne et ailleurs jusqu'à la prise en compte du diaconat par le Concile en 1965. Il montre que, dès l'origine, les deux possibilités de compréhension - suppléance des prêtres et degré original du sacrement de l'ordre - vont coexister, engendrant sans doute les difficultés que Daniel Moulinet et Denis Pelletier pointent dans leurs contributions.

Daniel Moulinet, pour sa part, aborde plus précisément les travaux du Concile, et leur environnement. Un exposé exhaustif présente la succession des travaux, et fait regretter une mise en œuvre thématique, dont on n'a qu'un bref aperçu dans la conclusion (une page) : une certaine frilosité théologique des Pères conciliaires, qui ne sera sans doute pas étrangère aux débats qui suivront.

7 L'historien Denis Pelletier présente les premières années du diaconat en France (1966-1976), à partir d'une base de données qu'il déplore limitée: des écrits pour l'essentiel originaires des milieux lyonnais, et pas de témoignages publics. Son intervention est articulée classiquement en trois points: les acteurs et étapes de l'introduction, les enjeux et débats suscités, l'épreuve de l'expérience. Il existe d'abord une offre de diaconat: des laïcs et des prêtres forment dès 1965 des groupes de candidats «spontanés ». En 1967, se crée un Comité national du Diaconat. En vis-à-vis une demande prudente se manifeste à partir du rapport de Mgr Mouisset, en 1965. L'épiscopat français attend les Motu proprio de 1967 et 1972, et peu d'évêques se lancent dans l'ordination de diacres. Appuyant et justifiant sans doute le piétinement de la hiérarchie, les débats portent dès le départ sur les risques de déstabilisation que cette figure ministérielle nouvelle produirait. Une inquiétude perceptible des prêtres se fait jour, qui porte aussi bien sur leur rôle que sur la mission des laïcs. Ils y voient un risque de démobilisation, et un niveau hiérarchique supplémentaire. Des laïcs leur font écho. La figure du diacre est une figure concurrente. En contrepoint, les discours défendant le diaconat empruntent à l'utopie de la rénovation en lieu et place de l'innovation, ce qui conduit à placer le diacre en figure médiatrice. Dans cette période, la charge d'utopie dans les débats est élevée. Cela explique-t-il le nombre relativement faible d'ordinations en France, de 1970 à 1976 ? On comptait, à cette date, trente-cinq diacres, contre trois cent dix en RFA et une centaine en Belgique. Denis Pelletier préfère conclure que ce piétinement et ces hésitations ont permis d'enraciner l'idée du diaconat, et préparé le terrain à une accélération des ordinations dans les années ultérieures. 
8 Après l'histoire, les approches sociologiques forment le corps de la deuxième partie. Elle est constituée de trois contributions majeures. D'une part une perspective de géographie sociale, présentée par Jean-René Bertrand et Colette Muller, qui avaient déjà cosigné Où sont passés les catholiques? (Paris, Desclée de Brouwer, 2002) déployant ce type de regard. Ensuite une vaste enquête sur les diacres, menée et analysée par Philippe Portier et Yoann Abiven, ouvre la question de la modernisation de l'Église par les diacres. Enfin, Céline Béraud croise la question de la modernisation par les diacres avec celle des femmes, à la fois comme laïques engagées et en tant qu'épouses des diacres.

9 À travers la répartition géographique des diacres (ordinations, périodes, lieux d'enracinement), se dégage-t-il un schéma d'intelligibilité de la gestion institutionnelle du catholicisme? Pour Jean-René Bertrand et Colette Muller, «la constitution de ce corps de ministres ordonnés se réalise progressivement par agrégation de nouveaux membres, par diffusion générale de l'appel au ministère» (p. 91). La densité démographique ne semble pas une clé de lecture pertinente (inégale répartition), ni la « contagion » locale. Des diocèses ne comptent que très peu de diacres, à côté d'autres qui en comportent beaucoup. Les indices de comportement religieux (lecteurs de la presse catholique, mouvements charismatiques, etc.) ne fournissent pas plus d'informations. Les diacres sont mariés ( $88 \%$ ), appartiennent aux catégories sociales aisées, sont ordonnés à cinquante ans (âge moyen depuis quarante ans). On ne peut ici résumer la très riche enquête analysée par les auteurs. Il en ressort cependant qu'aucune ligne claire en matière d'activités et de mission ne se dégage, signant peutêtre par là la fragilité des origines. Au vu du paysage décrit, on peut se demander toutefois s'ils ne formeraient pas un nouveau patriciat.

10 P. Portier et $Y$. Abiven présentent une analyse de l'enquête menée conjointement avec J.-R. Bertrand et C. Muller. Ils l'abordent sous l'angle de la modernisation telle que Peter Berger en a déployé les stratégies. Dans un monde capitaliste-individualiste, les institutions religieuses sont confrontées à la démonopolisation de leurs activités. Elles mettent alors en œuvre deux types de stratégies: résistance, c'est-à-dire finalité identique, mais adaptation pratique (pastorale); ou bien accommodement, avec modification substantielle des principes doctrinaux. La restauration du diaconat rentre bien dans le cadre de la première. Les diacres sont alors qualifiés de "passeurs de mondes » : " clerc parmi les hommes, il témoigne, par son état de ministre ordonné, de la transcendance à l'extérieur du sanctuaire. Homme parmi les clercs, il présentifie à l'autel, du fait de sa contribution aux affaires séculières, les souffrances, les appels des mondes où il est implanté » (p.135). Leur «indice de catholicité est très largement supérieur à celui de la moyenne nationale » (p.136). Ils disposent donc d'un capital d'ecclésialité laïque, que vient compléter un capital de sécularité (ils sont largement plus militants que la moyenne de leur catégorie sociale). Du reste, plus de $50 \%$ d'entre eux ont ressenti à un moment de leur vie, l'appel de la vocation sacerdotale. Ils restent cependant respectueux des prêtres, qu'ils souhaitent à plus de $55 \%$ voir conserver leur monopole de gestion sacramentelle, et à $21 \%$ d'apporter au monde la parole officielle de l'Église. Il se dégage alors deux profils de diacres : « les diacres "priants", d'un côté, insistent sur l'attestation identitaire (...) Les diacres "engagés", de l'autre, davantage portés au dialogue avec la culture moderne » (p.166). Au vu de la très riche analyse présentée dans ces pages, on s'interroge malgré tout sur l'espace que les diacres se sont créé peu à peu, entre les prêtres, quelque peu «refoulés» dans la gestion sacrale 
(l'image est sans doute un peu durcie), et les laïcs. L'ordination les installe en effet dans un rapport au temps long dont ne disposent pas ces derniers. Quel terrain institutionnel préparent-ils, à long terme?

11 Céline Béraud se penche pour sa part sur le cas des épouses de diacres, ainsi que sur la relation entre les diacres et les laïcs chargés de mission, très majoritairement des femmes. Deux dimensions, donc. Le statut d'époux procure aux diacres une assise issue de l'expérience, qui les fonde mieux en légitimité que l'appartenance institutionnelle. L'épouse du diacre est ainsi en second rôle, et contribue par sa présence à modifier le retentissement de la fonction. La contrepartie est une fracture instaurée dans le couple, puisque les fidèles ne voient plus que le diacre et sa femme. Bien que Céline Béraud ne le mentionne pas, on connaît des situations où les diacres ont vu leurs amis déserter leur foyer par peur d'une "contagion"; les femmes ne veulent pas que leur mari revête la robe liturgique. Les femmes ayant suivi des formations théologiques sont parfois plus compétentes, sur ce point, que les diacres. Il en résulte une compétition de légitimité : «[l'une] fondée sur la connaissance, dont se prévalent ces femmes (...), l'autre sur la sacralité que confère le sacrement de l'ordination » (p. 182). Charisme de fonction, en concurrence avec la compétence et la spécialisation des femmes laïques : n'est-ce pas une stratégie risquée, demande C. Béraud?

12 L'interrogation est également portée par Bernard Sesboüé, jésuite et théologien reconnu, qui s'appuie toutefois sur la théologie pour interroger la place des diacres. Son intervention avait ouvert le colloque. Elle emprunte à la forme scolastique de la disputatio: question, réponse positive, arguments contraires, position négative de l'auteur, arguments de l'auteur. Trois raisons d'abord de dire que l'expérience du diaconat est réussie. D'une part, leur nombre devient significatif (mille huit cents en France, vingt-cinq mille dans le monde en 2004). Corollairement, ils sont repérables et installés dans le paysage moderne, car ils sont des clercs mariés. Enfin, ils sont indispensables à la structure institutionnelle. (Ces trois arguments sont d'ordre sociologique, et non théologique). «Sed Contra » ensuite : le diaconat actuel n'est pas théologiquement assis sur trois références scripturaires majeures : Ac 6, Jn 13 et Trad. Apost. d'Hippolyte, 8. L'auteur souligne comment la Commission théologique internationale (CTI) a même pris soin de contourner les premier et troisième textes. « Respondeo » enfin : cinq raisons de déclarer que le diaconat n'a pas trouvé une assise solide dans la vie de l'Église catholique : 1 . Trente ans ne font pas le poids après mille ans d'occultation. Ce millénaire montre que l'on peut se passer des diacres, pas des prêtres ni des évêques... 2. Deux centres de gravité se disputent l'assise du diaconat: a) le service de la charité et de l'assistance, contre b) le diaconat est un degré du sacrement de l'Ordre. Ce dernier point est la raison de la disparition des diacres au Haut Moyen Âge. 3. Vatican II a déplacé les catégories de compréhension : «Vatican II est passé du sacerdoce comme catégorie-sujet du sacrement de l'ordre, depuis le Moyen Âge à Trente et dans les Temps modernes, à une catégorie-attribut pour les évêques et les prêtres (...) La catégorie mère qui rend compte du sens et des tâches du ministère ordonné n'est plus le sacerdoce mais l'envoi en mission par le Christ» (p. 201). Il est donc possible d'introduire le diaconat comme sacrement fondé sur le service et non sur le sacerdoce. Mais la théologie catholique récente a des réticences à déployer cette nouveauté, pourtant attestée dans les textes anciens. 4. L'émergence de laïcs avec lettres de missions, responsables de secteurs de la pastorale (le gouvernement pratique) introduit une confusion avec les diacres, que Rome ne semble pas vouloir lever. 5. Enfin, la relation entre sacrement du mariage et diaconat n'est pas explicitée. 
En résumé, le théologien souligne une certaine faiblesse de l'assise théologique de la réinstauration du diaconat. Il rejoint par là ce qui a été mis en évidence par les historiens.

Roland Minnerath, évêque de Dijon, dans l'étude sur la théologie du diaconat aux origines de l'Église, accrédite cette position. En listant six caractéristiques du diaconat contemporain (ordre à finalité propre ; exercice par des hommes mariés ; sacramentel ; diacre ministre ordinaire du baptême, assistant aux mariages et homéliaste; nature proche du sacerdoce, ou au contraire éloignée; origine théologique imprécise), il montre combien il s'éloigne de l'expérience des premiers siècles. Au passage, il offre une précieuse mise en garde aux chercheurs : le Catéchisme de l'Église catholique modifie ses contenus au fur et à mesure de ses éditions! (p. 213). Sa conclusion ouvre quelques pistes aux théologiens pour reprendre l'articulation entre tradition et innovation.

Didier Gonneaud, théologien à la faculté de théologie de l'université catholique de Lyon, et auprès $d u$ Comité national du Diaconat, propose pour sa part une confrontation entre le code de droit canonique et les textes de Vatican II. Il conclut que les diacres n'appartiennent pas à un collège, et donc n'ont pas la responsabilité de l'unité des tria munera (trois «fonctions») caractéristiques du sacerdoce: enseigner, sanctifier, gouverner. Alphonse Borras, professeur de droit canonique à l'université catholique de Louvain-la-Neuve, lui emboîte le pas, et précise l'articulation entre l'épiscopat, en tant qu'ordre lié au presbytérat, et le diaconat qui met en œuvre une potestas juridique à partir des missions données par l'épiscopat. Jean-François Chiron, doyen de la faculté de théologie de Lyon, s'efforce de problématiser théologiquement la distinction entre diaconat et presbytérat, sans toutefois nier que les circonstances pratiques (effondrement du clergé presbytéral) puissent déterminer l'approche ecclésiologique elle-même.

15 La quatrième partie est consacrée à des réflexions et témoignages. Nous n'en mentionnerons que quelques-unes. Alphonse Borras, déjà cité, souligne les hésitations de l'institution ecclésiale pour trouver une place cohérente aux diacres en Belgique. Francis Deniau, évêque de Nevers, retrace son expérience positive du diaconat et conclut sur des mises en garde pour le laisser se déployer harmonieusement. Henri Denis, théologien et ancien expert au concile Vatican II, porte un regard optimiste, en soulignant les potentialités ecclésiales du diaconat. Une seule épouse de diacre apporte son témoignage, sous la forme d'un résultat d'enquête menée auprès des épouses de diacres dans la région Nord.

Le colloque a donc ouvert des questionnements importants, et l'interdisciplinarité se justifie ici pleinement. Les interrogations des théologiens sont susceptibles d'être transcrites en termes sociologiques, tandis que les questionnements sociologiques trouvent des échos dans la théologie. L'histoire n'est pas en reste, ouvrant une interrogation sur la fécondité de la mémoire, aussi bien celle de long terme (nous l'avons vu avec la comparaison entre diaconat des origines et l'actuel) que celle de court terme (avec les hésitations sur la catégorie fondatrice du diaconat: service ou ministère cultuel ?) Tout au plus regrettera-t-on la faible place faite aux autres acteurs sujets de l'inconfort diaconal : les laïcs animateurs de communautés ou responsables de services, les femmes, les prêtres, qui semblent bousculés par l'irruption de ces nouveaux acteurs... ce serait vraisemblablement un autre colloque.

$17 \mathrm{Au}$ total, ce livre constitue un repère intéressant dans l'auto-évaluation d'une institution, peut-être en déclin (en France), mais soucieuse de répondre 
structurellement aux inquiétudes qu'elle perçoit. L'avenir dira si la réponse par le diaconat est à la hauteur du défi posé par la Modernité. 